\begin{tabular}{|c|l|}
\hline Title & Stretching-induced ion complexation in physical poly ampholyte hydrogels \\
\hline Author(s) & $\begin{array}{l}\text { Cui, Kunpeng; Sun, Tao Lin; Kurokawa, Takay uki; Nakajima, Tasuku; Nonoyama, Takay uki; Chen, Liang; Gong, Jian } \\
\text { Ping }\end{array}$ \\
\hline Citation & $\begin{array}{l}\text { Soft matter, 12(43), 8833-8840 } \\
\text { https://loi.org/10.1039/c6sm01833e }\end{array}$ \\
\hline Issue Date & $2016-11$-21 \\
\hline Doc URL & http://hdl.handle.net/2115/67764 \\
\hline Type & article (author version) \\
\hline File Information & Stretch-induced ion complexation.pdf \\
\hline
\end{tabular}

Instructions for use 


\title{
Stretch-Induced Ion Complexation in Physical Polyampholyte Hydrogels
}

\author{
Kunpeng Cui ${ }^{1}$, Tao Lin Sun ${ }^{1,2}$, Takayuki Kurokawa ${ }^{1,2}$, Tasuku Nakajima ${ }^{1,2}$, Takayuki \\ Nonoyama $^{1,2}$, Liang Chen ${ }^{3}$, Jian Ping Gong ${ }^{1,2^{*}}$ \\ ${ }^{1}$ Faculty of Advanced Life Science, Hokkaido University, Sapporo 060-0810, Japan \\ ${ }^{2}$ Global Station for Soft Matter, Global Institution for Collaborative Research and Education, \\ Hokkaido University, Sapporo, Japan \\ ${ }^{3}$ Graduate School of Life Science, Hokkaido University, Sapporo, Japan \\ *Corresponding author: gong@mail.sci.hokudai.ac.jp
}

\begin{abstract}
Recently, we have developed a series of charge balanced polyampholytes (PA) physical hydrogels by random copolymerization in water, which show extraordinarily high toughness, self-healing ability and viscoelasticity. The excellent performance of PA hydrogels is ascribed to the dynamic ionic bonds formation through inter- and intra-chain interactions. The randomness makes ionic bonds of a wide strength distribution, the strong bonds, serve as permanent crosslinking, imparting the elasticity, while the weak bonds reversibly break and re-form, dissipating energy. In this work, we developed a simple physical method, named as pre-stretch method, to promote the performance of PA hydrogels. By imposing a pre-stretch on the sample at as-prepared state, the ion complexation during dialysis is prominently accelerated and the final performance is largely promoted. Further analysis suggests that the strong bonds formation induced by pre-stretch is responsible for the change in final performance. Pre-stretch decreases the entropy of system and increases the chain alignment, resulting in an increased possibility for strong bonds formation.
\end{abstract}




\section{Introduction}

Hydrogels consisted of network structure and abundant of water are typically soft and wet materials, which bear analogy to biological soft tissues and show great potential for use as biomaterials to substitute damaged real ones ${ }^{1-4}$. However, conventional hydrogels are soft, weak and brittle, in contrary to the robust and tough biological tissues ${ }^{5-7}$. To address this problem, double-network hydrogels (DN gels) were developed ${ }^{8-11}$. This new class of gels is mechanical hard, strong and comparable to cartilages even contain 80 to 90 weight percent of water. DN gels are consisted of two interpenetrated polymer networks with strong asymmetric structure. The first network is tightly cross-linked and highly stretched, making it stiff and brittle, which serves as sacrificial bonds and dissipates energy. The second network is loosely cross-linked and flexible, making it soft and stretchable, which acts as hidden length to sustain large deformation.

The toughing of DN gel is attributed to the irreversible broken of covalent bonds in the first network, making it softening by large deformation and limited practical application. However, in principle, the DN concept can be generalized to self-healing materials with reversible bonds rather than covalent bonds as sacrificial bonds. Along this line, several tough and self-healing hydrogels were successfully developed ${ }^{12-15}$. For example, Sun et al. synthesized a stretchable and tough hydrogel with ionically crosslinked alginate as sacrificial bonds ${ }^{15}$ and Chen et al. developed a tough and fatigue resistant hydrogel with hydrogen bond as sacrificial bonds ${ }^{12}$. Recently, we reported a new class of charge balanced linear polyampholytes (PA) physical hydrogels by random copolymerization, which show extraordinarily high toughness, high fatigue resistance, self-healing ability and 
viscoelasticity $^{16,17}$. In PA hydrogels, multiple ionic bonds, formed through intra- or interchain interactions, show a wide distribution in strength due to random nature of charges. The ionic interactions can be categorized into two groups, strong bonds and weak bonds, differ in number of ionic pairs. The former serves as quasi-permanent crosslinks, to sustain the elasticity, while the latter breaks and reforms reversibly, to dissipate energy.

In this work, we aim to tailor the performance of physical PA hydrogels with simple physical method. To reach this purpose, a pre-stretch is imposed to the as-prepared P(NaSSco-MPTC) hydrogels composed of two oppositely charged ionic monomers, anionic monomer p-styrenesulfonate (NaSS) and cationic monomer 3-(methacryloylamino) propyltrimethylammonium chloride (MPTC) at optimized composition. According to our previous work $^{17}$, only a few ionic interactions between NaSS and MPTC exist in as-prepared PA samples because of the existence of large amount of counter ions. The ionic interactions mainly formed during dialysis process as the remove of counter ions. We conjecture that the pre-stretch can enhance strong bonds formation and thus influence the final mechanical performance. Because to form strong bonds, the adjacent chains need to align and parallel to each other, which is difficult for polymer chains in random coil conformation (Road II, Scheme 1). While pre-stretch can induce chain alignment, and thus largely increase the possibility of strong bonds formation (Road I, Scheme 1). Our results support this conjecture and demonstrate that both the ion complexation during dialysis process and performance at final state are influenced by the pre-stretch. Compared to the existing methods, such as changing chemical component or adding fillers (glass fiber or nanocomposite), this newly developed pre-stretch method is simple, harmless, economic and can change the performance 
of hydrogels effectively. The approach developed here may applicable to other physical hydrogel systems.
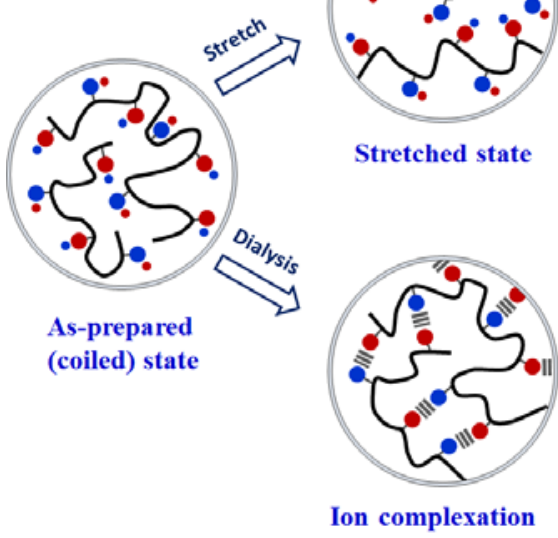

Stretched state

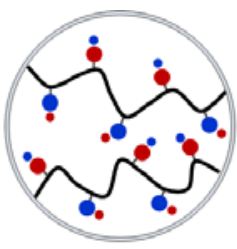
Dialysis $\stackrel{\text { Dialysis }}{\longrightarrow}$

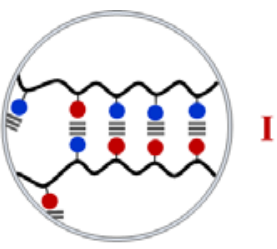

Ion complexation

Positively

charged group

Negatively

- Counterions

Scheme 1. Schematic illustration of dialysis process of PA hydrogels. (I) Dialysis with prestretch, which promotes strong bonds formation due to the chain alignment induced by prestretch. (II) Dialysis without pre-stretch.

\section{Experimental section}

Synthesis of PA hydrogels. The one-step random copolymerization procedure of PA hydrogels here is same as that in our previous work ${ }^{16,17}$. In brief, a mixed aqueous solution of NaSS, MPTC and UV initiator $\alpha$-ketoglutaric acid was injected into a sandwich mold consisted of two glass plates and a $2 \mathrm{~mm}$ thickness silicone spacer, and then irradiated with $365 \mathrm{~nm}$ UV light for 11 hours at argon atmosphere. An optimized composition with total monomer concentration $C_{\mathrm{m}}$ of $2.1 \mathrm{M}$, molar ratio of $\mathrm{NaSS}: \mathrm{MPTC}=0.525: 0.475$, and 0.25 mol $\% \alpha-$ ketoglutaric acid relative to $C_{\mathrm{m}}$ was used. All the materials, including NaSS, MPTC and $\alpha-$ ketoglutaric acid were pursued from Wako Pure Chemical Industries, Ltd. (Japan) and used as received. The water used in all experiments was millipore deionized. 
Pre-stretch. A simple homemade extension device was used to impose pre-stretch on samples. Four states are defined here (Figure s1), as-prepared state, stretched state, equilibrium state and free state. The as-prepared hydrogels (as-prepared state) were firstly fixed between two clamps of the extension device. Then the samples were stretched uniaxially along length direction (stretched state). The length, width and thickness of sample at as-prepared state were $20 \mathrm{~mm}, 50 \mathrm{~mm}$ and $2 \mathrm{~mm}$, respectively. After pre-stretch, the length and width of sample changed to $L_{1}$ and $W_{1}$, respectively. The pre-stretch ratio is defined as $\lambda_{\text {pre }}=L_{1} / L_{0}$, here $L_{0}=20 \mathrm{~mm}$ is the initial length at as-prepared state. The pre-stretched samples were further immersed into large amount water to dialyze longer than one week to reach equilibrium state. The change of sample width during dialysis process was recorded. The final width swelling ratio is defined as $Q=W_{2} / W_{1}$, where $W_{2}$ is sample width at equilibrium state. After reaching equilibrium, the samples were released from extension device to free state, accompanying with the length changed from $L_{1}$ to $L_{3} . L_{3}$ was measured after release for 48 hours. Compared to the initial as-prepared state, a fixed stretch ratio exists at free state, which is defined as $\lambda_{\text {fixed }}=L_{3} / L_{0}$.

Tensile test. The uniaxial tensile stress-strain measurements were performed in moisture environment using a commercial tensile tester (Tensilon RTC-1310A, Orientec Co.). The free state samples with thickness between 1-2 mm thickness were cut into dumbbell shape with the standard JIS-K6251-7 size (length $12 \mathrm{~mm}$ and width $2 \mathrm{~mm}$ ). The stress was calculated with the cross-section at free state. The Young's modulus was calculated from initial liner region of stress-strain curve (with strain smaller than 0.15 ). The fracture stress and strain are defined as the nominal stress and strain at breaking point. The work of 
extension at fracture $W_{\text {extf }}$ is defined as the area below stress-strain plot. Loading-unloading test was also conducted at same stretch velocity and sample size. The sample was firstly stretched to a strain $\varepsilon=4$, then returned back to the initial displacement with same stretch velocity immediately. The hysteresis ratio is defined as the area ratio of the area between loading and unloading stress-strain curve to the area under loading stress-strain curve. All the tensile test was performed at room temperature and under constant stretch velocity 100 $\mathrm{mm} \min ^{-1}$.

Wide-angle X-ray scattering (WAXS) measurement. WAXS measurement was performed for the free state samples. A Rigaku X-ray crystallography device equipped with $\mathrm{Cu}$ radiation was used (wave-length of $0.154 \mathrm{~nm}$ ). The voltage and current of X-ray generator were $40 \mathrm{KV}$ and $30 \mathrm{~mA}$, respectively. Sample to detector distance was set to $84.5 \mathrm{~mm}$ and exposure time for each sample was 1 hour. 


\section{Result and Discussion}
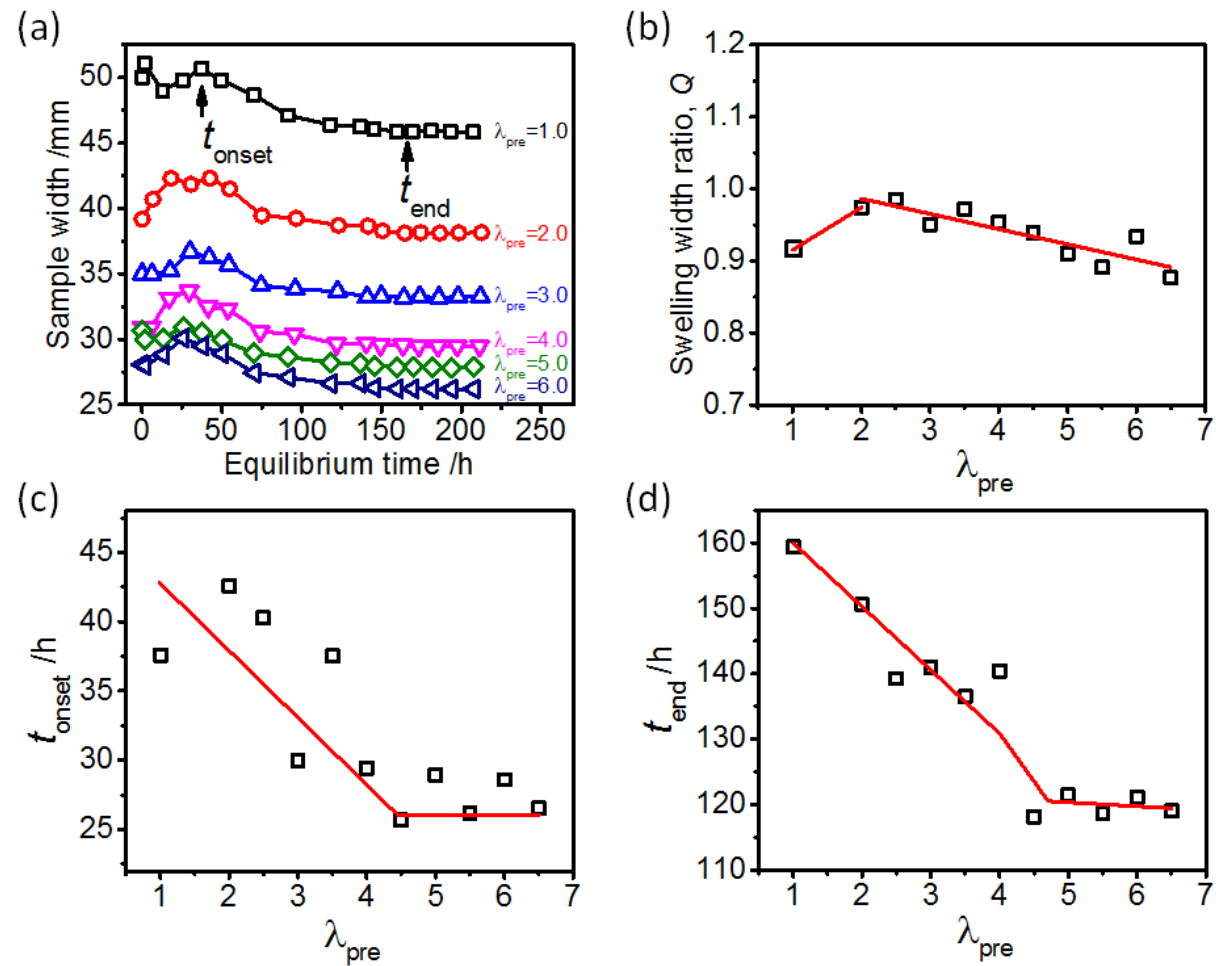

Figure 1. (a) Width changes with time during dialysis process of samples with different prestretch ratio. (b) Evolution of final width swelling ratio with pre-stretch ratio. (c) The onset time and (d) the end time change with pre-stretch ratio.

First, we present how pre-stretch influence the dynamics of ion complexation process. From our previous study ${ }^{16,17}$, PA hydrogels at current experimental conditions show shrinkage as a result of formation of ion complex structure. As it is difficult to detect the ion complexation directly, the sample width was chosen as a parameter to characterize this process. During dialysis process, for all PA hydrogels with different pre-stretch ratio, the sample width first increases with equilibrium time, then decreases, and finally reaches a plateau (Figure 1a). This non-monotonic evolution of sample width is consistent with the reported results of English et al. ${ }^{18}$, which may be caused by the small ions in the gel that 
diffused into water during the dialysis. But the exact reason is still unclear due to the dynamic and complicated nature of this process and further study is needed. Here we define two parameters, $t_{\text {onset }}$ and $t_{\text {end }}$ to quantify how pre-stretch influence the ion complexation process. $t_{\text {onset }}$ and $t_{\text {end }}$ are the time when sample width reaches largest peak value and initial plateau, respectively. As shown in Figure 1c and 1d, both $t_{\text {onset }}$ and $t_{\text {end }}$ first decrease with increasing pre-stretch ratio, then saturate at pre-stretch ratio of about 4.5. This result indicates that the ion complexation process indeed can be greatly accelerated by imposing a simple pre-stretch. Accelerated ion complexation process means short production time. Thus this method may be promising and economic for future industrialization of PA hydrogels.

Figure $1 \mathrm{~b}$ depicts the final swelling width ratio $(Q)$ at stretch state as a function of pre-stretch ratio $\lambda_{\text {pre }}$. Gels at all pre-stretch ratios shrunk $(Q<1)$ and $Q$ decreases with $\lambda_{\text {pre }}$ for $\lambda_{\text {pre }}>1$, in contrast to the nonionic chemical hydrogels where swelling ratio increases with stretch ratio ${ }^{19-22}$. The shrinkage or swelling equilibrium of PA hydrogels is determined by the balance among following three factors, isotropic mixing $\left(\Delta F_{\text {mix }}\right)$ between network and water to swell the network, ion complexation $\left(\Delta F_{\text {ion }}\right)$ to contract the network and the elastic energy $\left(\Delta F_{\text {el }}\right)$ due to the deformation of the network ${ }^{16-18}$. The contributions of those three factors to the free energy $(\Delta F)$ are assumed to be additive, $\Delta F=\Delta F_{\text {mix }}+\Delta F_{\text {ion }}+\Delta F_{\mathrm{el}}$. For nonionic hydrogels $\left(\Delta F_{\text {ion }}=0\right), \Delta F_{\text {el }}$ increases with stretch ratio, leading to an increase of swelling ratio. For PA hydrogels studied here, if the ion complexation is not influenced by pre-stretch ( $\Delta F_{\text {ion }}$ keeps constant), $Q$ should also increase with $\lambda_{\text {pre }}$ as observed in nonionic hydrogels. The $Q$ decreases with $\lambda_{\text {pre }}$ further demonstrates pre-stretch can enhance the ion complexation process. At small $\lambda_{\text {pre, }}$, the increase of $\Delta F_{\text {ion }}$ by pre-stretch is smaller than that 
of $\Delta F_{\mathrm{el}}$. So $Q$ for small $\lambda_{\text {pre }}$ is a little bit larger than that for un-stretched sample $\left(\lambda_{\text {pre }}=1\right)$. But $\Delta F_{\text {ion }}$ may increase much faster with $\lambda_{\text {pre }}$ than $\Delta F_{\text {el }}$, that can account for $Q$ decreases with $\lambda_{\text {pre }}$
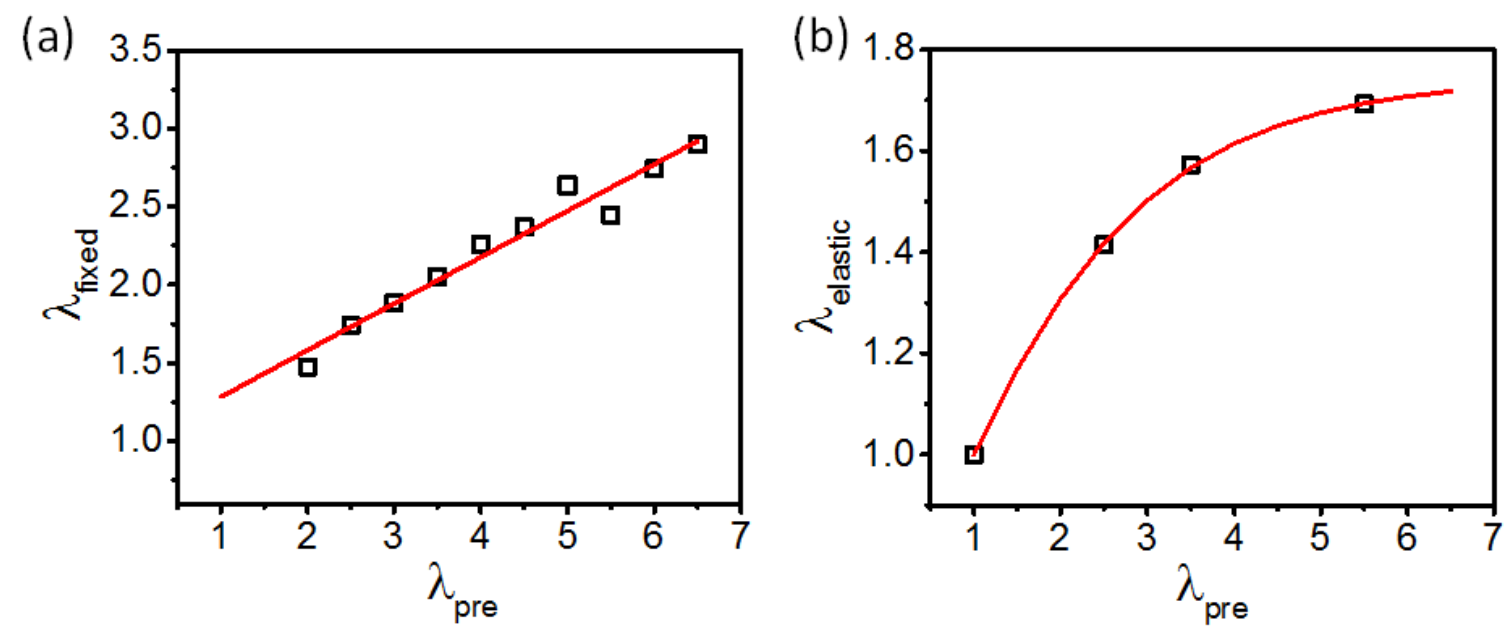

Figure 2. (a) The fixed stretch ratio $\left(\lambda_{\text {fixed }}\right)$ at free state and (b) contribution of elastic part ( $\lambda$ elastic) as a function of pre-stretch ratio.

After reaching equilibrium of dialysis, samples were released from stretched state to free state. The pre-stretched sample shrunk in length direction but could not totally return back to the initial un-stretched state, i.e., a fixed stretch ratio $\lambda_{\text {fixed }}$ exists. $\lambda_{\text {fixed }}$ increases almost linear with $\lambda_{\text {pre }}$ as shown in Figure 2a. Two possible reasons are responsible for the $\lambda_{\text {fixed }}$, chain orientation or chain flow (creep effect) or both. To check whether chain orientation contributes, WAXS measurement was conducted for samples at free state (Figure S3 and S4). WAXS results demonstrate that samples have a weak orientation and the orientation degree increases with increasing $\lambda_{\text {pre }}$ (Figure S4), indicating that chain orientation contributes to $\lambda_{\text {fixed }}$. To further confirm whether chain flow exists, samples at free state were immersed in $0.2 \mathrm{~mol} / \mathrm{L}$ sodium chloride solution for one week (Figure S5). 
For un-stretched case, the sample size keeps almost constant. But for the pre-stretched sample, shrinkage in pre-stretch direction can be observed. This is because the introduction of counter ions destroys the ion complex structure induced by pre-stretch, leading to the swelling of samples. This result clearly indicates that the chain orientation is sustained by ion complex structures. The contribution of chain orientation $\left(\lambda_{\text {elastic }}\right)$ can be considered as the shrunken part and calculated based on the size change (Figure 2b). $\lambda_{\text {elastic }}$ increases with $\lambda_{\text {pre }}$ and reaches a maximum of about 1.7, much smaller than maximum of $\lambda_{\text {fixed }}$ (about 3.0), suggesting both chain orientation and chain flow contribute to the $\lambda_{\text {fixed }}$.

(a)

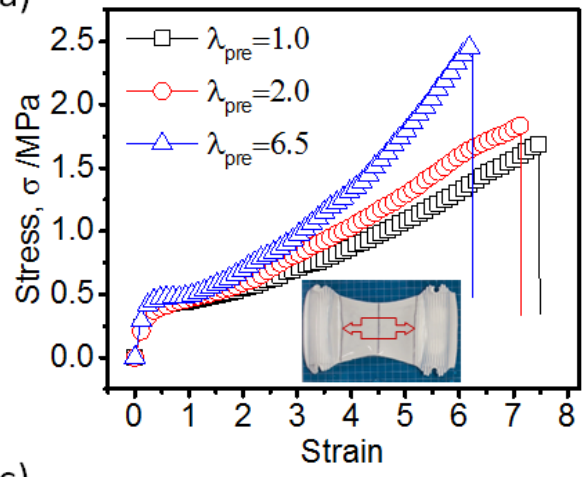

(c)

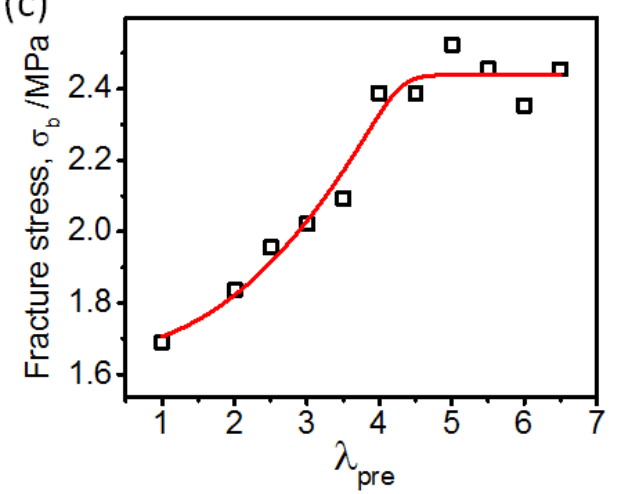

(b)

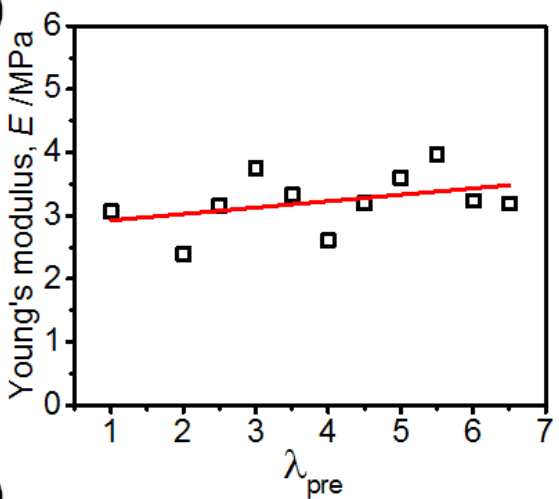

(d)

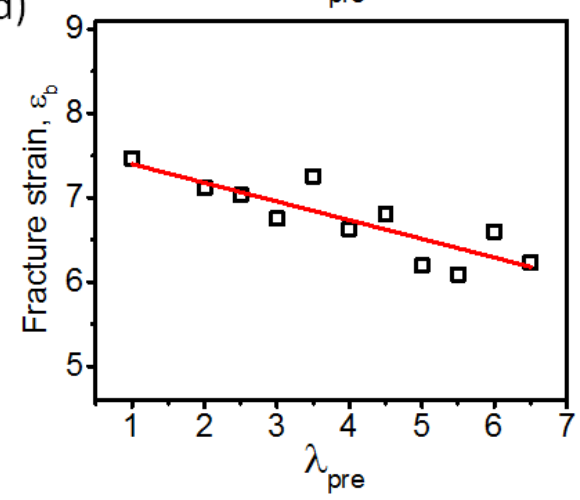

Figure 3. (a) Tensile behaviors of PA hydrogels along pre-stretch direction at different prestretch ratio in their free state. The insert shows an optical image of sample at free state. (b) Young's modulus $(E),(\mathrm{c})$ fracture stress $\left(\sigma_{\mathrm{b}}\right)$, and $(\mathrm{d})$ fracture strain $\left(\varepsilon_{\mathrm{b}}\right)$ as a function of prestretch ratio. 
The pre-stretch not only accelerates ion complexation process, but also influences the final property of hydrogels. Figure 3 presents the pre-stretch ratio dependence of tensile behavior of PA hydrogels along pre-stretch direction at the free state. The Young's modulus $(E)$ at studied pre-stretch range is around $3 \mathrm{MPa}$ and has a weak increase with increasing $\lambda_{\text {pre }}$, while the fracture stress is significantly increased from about 1.7 to $2.4 \mathrm{MPa}$, accompanying with a decrease in fracture strain. The fracture stress shows a two stage processes, first increases with $\lambda_{\text {pre }}$ and then reaches a plateau, the transition point of which is same as that

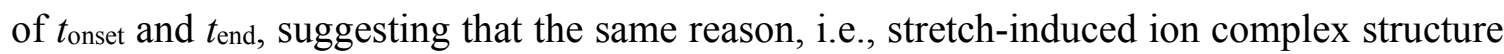
formation, is responsible for the change in both dynamics of ion complexation and final tensile behavior.
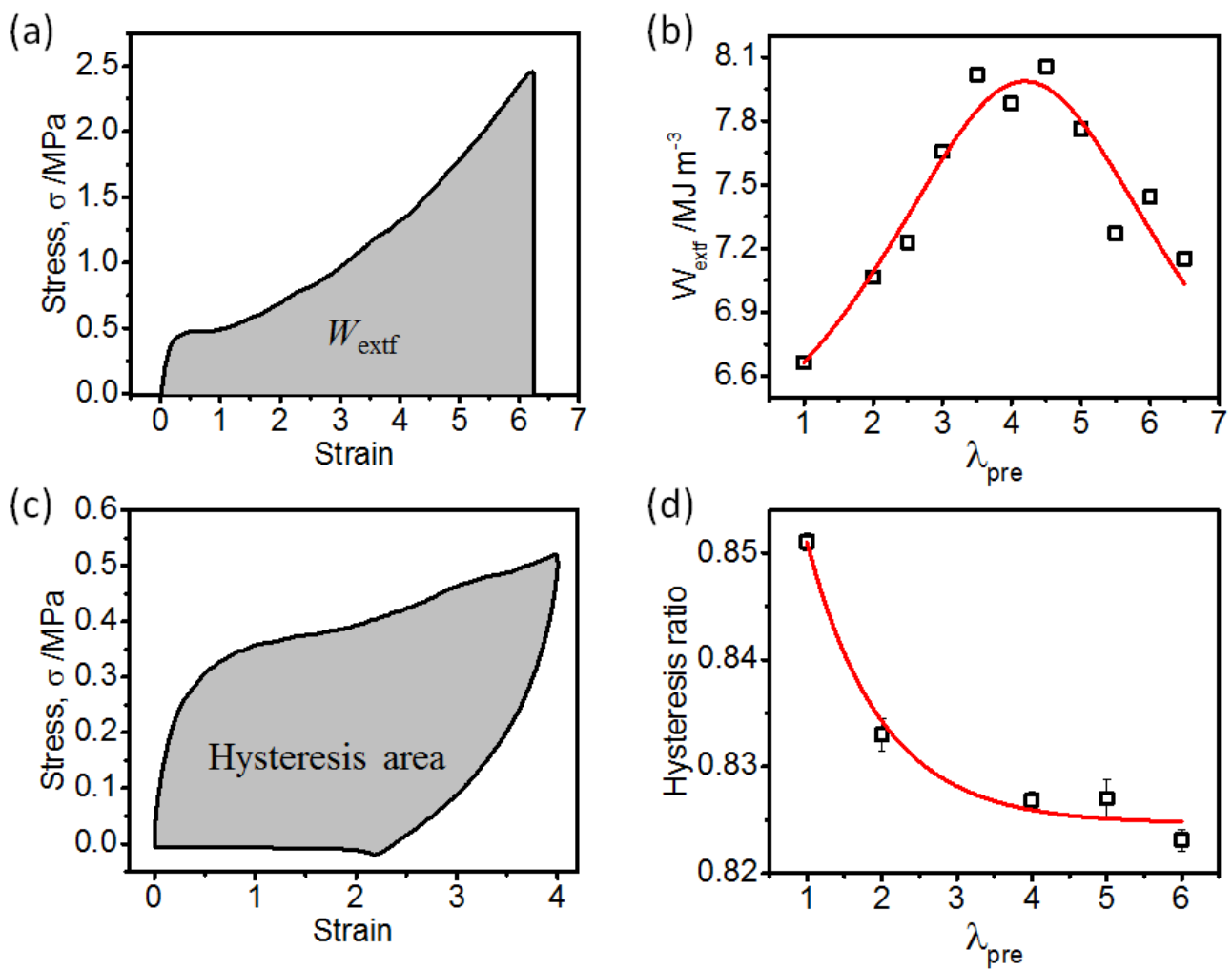
Figure 4. Examples show how to calculate the (a) work of extension at fracture ( $\left.W_{\text {extf }}\right)$ and (c) hysteresis area. Hysteresis area is used to calculate hysteresis ratio. Pre-stretch ratio dependence of (b) work of extension at fracture and (d) hysteresis ratio of PA hydrogels at the free state.

Based on stress-strain curves, the work of extension at fracture ( $\left.W_{\text {extf }}\right)$, a quantity to assesses the toughness of hydrogels, can be calculated as shown in Figure $4 \mathrm{a}$. For $\lambda_{\text {pre }}<3.5$, $W_{\text {extf }}$ increases with $\lambda_{\text {pre }}$. The largest values of $W_{\text {extf }}$ appears for samples with $\lambda_{\text {pre }}$ ranging from 3.5 to 4.5 . When $\lambda_{\text {pre }}>4.5, W_{\text {extf }}$ decreases with $\lambda_{\text {pre }}$, which is easily to understand because the fracture stress keeps almost constant while the fracture strain decreases (Figure 3). A large hysteresis presents in loading-unloading curve (Figure 4c), suggesting large amount energy were dissipated during deformation. While the hysteresis ratio decreases with $\lambda_{\text {pre }}$, implying the ion complex structure induced by pre-stretch suppresses the dissipation process.

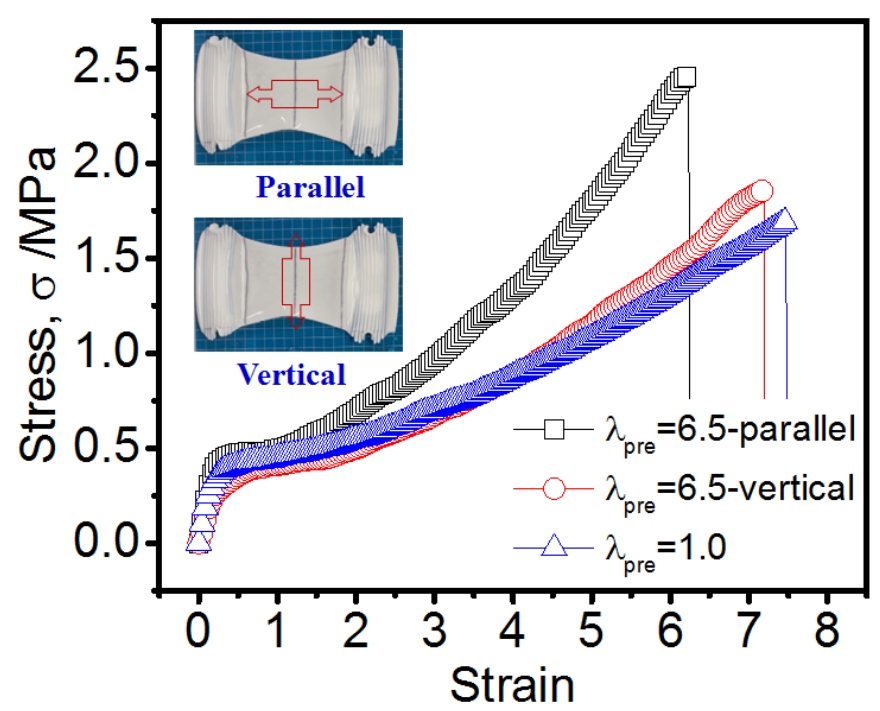

Figure 5. Stress-strain curves in directions parallel and vertical to pre-stretch of sample with pre-stretch ratio of 6.5 at the free state. For comparison, stress-strain curve of un-prestretched sample is also presented. The inserts indicate the stretch directions of tensile test. 
As demonstrated in WAXS measurement, samples with pre-stretch have a weak orientation, which can be reflected in stress-strain curves. Figure 5 gives a typical example of sample with pre-stretch ratio 6.5 to show the anisotropic mechanical performance. The fracture stress in pre-stretch direction is much larger than that in vertical direction, while the fracture strain is smaller. Interestingly, the performance in vertical direction is not weaker than the un-stretched sample. This is probably attributed to the weak orientation of polymer chains in network structure, leading to the similar performance in vertical direction to that of un-stretched sample. The ion complex structure induced by pre-stretch increases the performance in pre-stretch direction, but may contribute little to the vertical direction due to its preferred orientation, resulting in a large difference in performance in parallel and vertical directions.

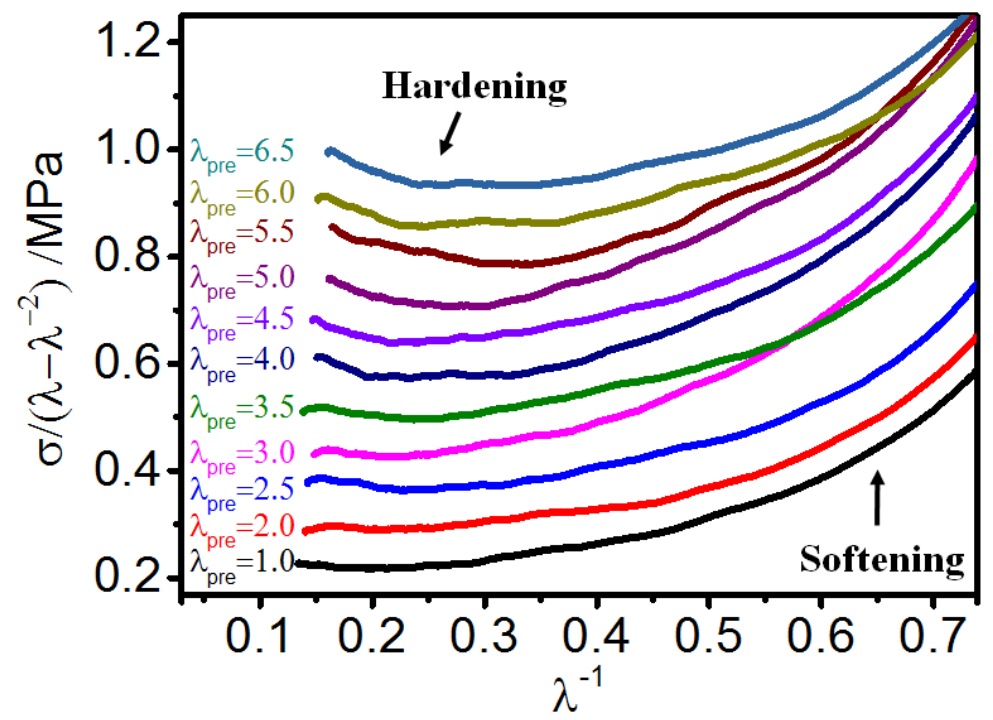

Figure 6. Mooney-Rivlin plots of PA hydrogels at different pre-stretch ratio in their free state. The plots are shifted along vertical direction to give a clearer visualization.

To better understand the ion complex structure induced by pre-stretch, the phenomenological Mooney-Rivlin equation is used to analyze the stress-strain relationship 
of PA hydrogels at different pre-stretch ratio. The Mooney-Rivlin equation can be expressed as follows ${ }^{17,23-26}$ :

$\sigma_{\text {red }}=\frac{\sigma}{\lambda-\lambda^{-2}}=2 C_{1}+2 C_{2} \frac{1}{\lambda}$

where $\sigma_{\text {red }}$ is the reduced stress, $C_{1}$ is material constant and equal to half of shear modulus, and $C_{2}$ is related to the strain hardening $\left(C_{2}<0\right)$ or softening $\left(C_{2}>0\right)$ beyond the Gaussian elasticity region. For all the samples with different pre-stretch ratio, the Mooney-Rivlin plots show strain softening at $\lambda^{-1}>0.4$ (Figure 6), suggesting the breakage of inter-chain ionic bonding. The strain softening is more prominently in samples with low pre-stretch ratio, indicating that more number of weak ion bonds were broken at low pre-stretch ratio during deformation. This result is consistent with loading-unloading curve in Figure 4, where sample at small pre-stretch ratio has larger hysteresis ratio. On the contrary, strain hardening is more prominently at high pre-stretch ratio. The strain hardening is attributed to finite extensibility of polymer chains at the presence of crosslinking or quasi-permanent crosslinking. So the more prominent strain hardening of samples at high pre-stretch ratio means more quasipermanent crosslinking formed as no chemical crosslinking exists. In our PA hydrogels, only strong ionic bonds can act as quasi-permanent crosslinking. That is to say, pre-stretch enhances the strong bonds formation. 

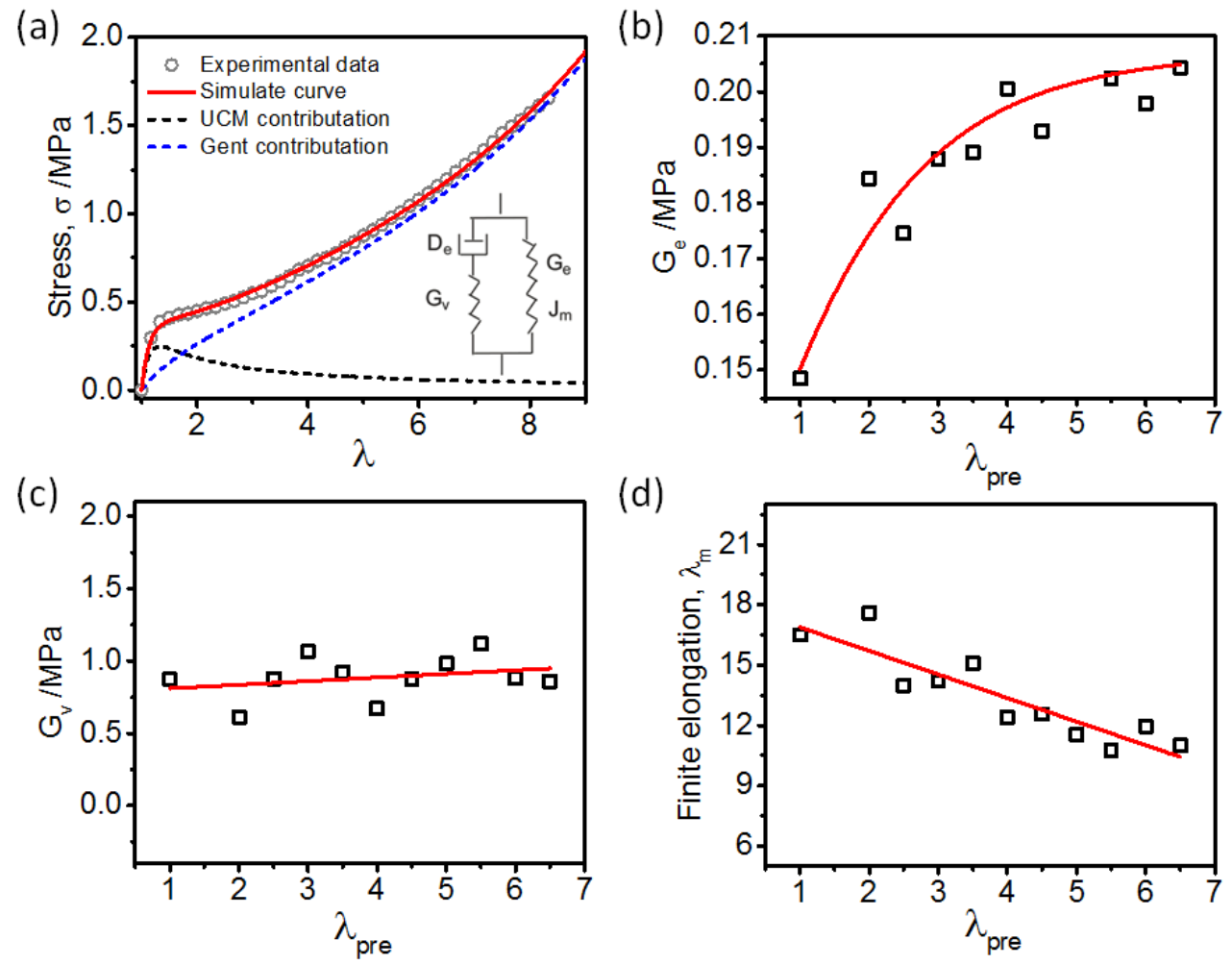

(d)

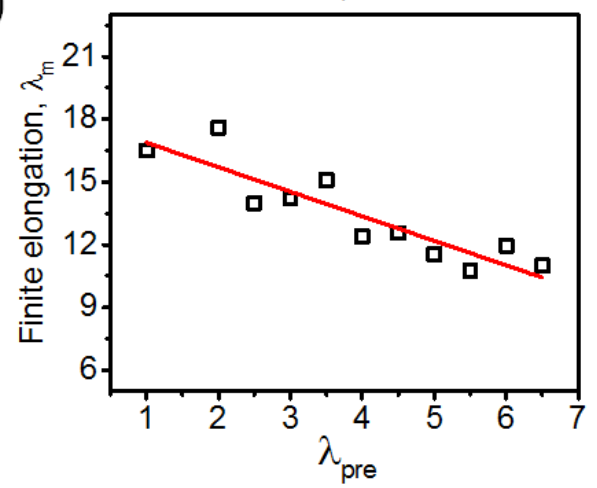

Figure 7. (a) Typical tensile behavior of PA hydrogels at the free state, the simulated curves of viscoelastic model, and the contributions of viscous part (UCM) and elastic part (Gent). The insert shows the schematic of the viscoelastic model. Pre-stretch ratio dependence of (b) shear modulus of elastic part $\mathrm{G}_{\mathrm{e}}$, (c) shear modulus of viscoelastic part $\mathrm{G}_{\mathrm{v}}$, and (d) theoretical finite elongation $\lambda_{\mathrm{m}}$.

To further validate above speculation, a viscoelastic model was used to simulate the tensile behavior. This viscoelastic model was proposed by Creton et al. ${ }^{27}$ and has been demonstrated well suitable for our PA system ${ }^{17}$. As shown in Figure $7 \mathrm{a}$, the model is consisted of two parallel parts, the Upper Convected Maxwell (UCM) model to describe viscoelastic flow and Gent hardening model to describe strain hardening due to finite extensibility. The nominal stress $\sigma_{\mathrm{N}}$ is expressed as the sum of UCM element $\sigma_{\mathrm{N}, \mathrm{v}}$ and Gent element $\sigma_{\mathrm{N}, \mathrm{e}}$ :

$\sigma_{\mathrm{N}}(\lambda)=\sigma_{\mathrm{N}, \mathrm{v}}(\lambda)+\sigma_{\mathrm{N}, \mathrm{e}}(\lambda)$ 
where the viscoelastic stress $\sigma_{\mathrm{N}, \mathrm{v}}$ is:

$$
\begin{array}{r}
\sigma_{\mathrm{N}, \mathrm{v}}(\lambda)=\left(\frac{2 G_{\mathrm{v}} D_{\mathrm{e}}}{1-2 D_{\mathrm{e}}}\left(1-\exp \left(-\frac{\left(1-2 D_{\mathrm{e}}\right)}{D_{\mathrm{e}}}(\lambda-1)\right)\right)\right. \\
\left.+\frac{G_{\mathrm{v}} D_{\mathrm{e}}}{1+D_{\mathrm{e}}}\left(1-\exp \left(-\frac{\left(1+D_{\mathrm{e}}\right)}{D_{\mathrm{e}}}(\lambda-1)\right)\right)\right) \lambda^{-1}
\end{array}
$$

and the entropic elastic stress $\sigma_{\mathrm{N}, \mathrm{e}}$ is:

$\sigma_{\mathrm{N}, \mathrm{e}}(\lambda)=\left(\frac{G_{\mathrm{e}}}{1-\frac{\lambda^{2}+2 \lambda^{-1}-3}{J_{\mathrm{m}}}}\left(\lambda^{2}-\lambda^{-1}\right)\right) \lambda^{-1}$

Here $G_{\mathrm{v}}$ and $G_{\mathrm{e}}$ are initial shear modulus of viscoelastic part and elastic part, respectively. According to our previous work ${ }^{17}, G_{\mathrm{v}}$ is ascribing to the breaking of weak bonds during deformation, while $G_{\mathrm{e}}$ is attributed to the contribution of strong bonds which act as quasipermanent crosslinking and do not flow during deformation. $D_{\mathrm{e}}$ is the dimensionless Deborah number and defined as the product of strain rate and relaxation time of viscous component. $J_{\mathrm{m}}$ is the maximum allowable value of the first strain invariant, which can be expressed as a function of theoretical finite extensibility of network chains $\lambda_{\mathrm{m}}$ :

$J_{\mathrm{m}}=\lambda_{\mathrm{m}}{ }^{2}+2 \lambda_{\mathrm{m}}{ }^{-1}-3$

This model has four parameters, $G_{\mathrm{e}}, G_{\mathrm{v}}, \lambda_{\mathrm{m}}$ and $D_{\mathrm{e}}$. As Young's modulus $E=3\left(G_{\mathrm{e}}+G_{\mathrm{v}}\right)$ can be calculated from stress-strain curve, the tensile behavior can be fitted with three independent parameters.

Figure 7a presents an optimized simulation results using eqn (2) for stress-elongation curve of hydrogels, where a nice fitting is observed between simulated results and 
experimental curve. Figure $7 \mathrm{~b}$ gives the $G_{\mathrm{e}}$ at various pre-stretch ratio $\lambda_{\text {pre }}$, which increases substantially by increasing $\lambda_{\mathrm{pre}} \cdot G_{\mathrm{e}}$ represents strong bonds, meaning that pre-stretch indeed can significantly increase strong bonds formation. This result is consistent with Mooney analysis and demonstrates our conjecture in Scheme 1. Interestingly, the $G_{\mathrm{e}}$ and the fracture stress (Figure 3c) show similar tendency with $\lambda_{\text {pre }}$, implying an intrinsic correlation exists between them. This is reasonable because strong bonds act as quasi-permanent crosslinking and would be fractured when the chain network reach finite extensibility. Our previous work has demonstrated that the ionic association energy of strong bonds is comparable to the covalent bond dissociation energy ${ }^{16}$. Therefore, the strong bonds should break near or at fracture strain and contribute to the fracture stress.

Different to strong bonds, the weak bonds represented by $G_{\mathrm{v}}$ only have a weak increase as demonstrated in Figure 7c. Pre-stretch enhances the possibility of chain parallel alignment and thus influences the formation of ion bonds. For strong bonds formation, multiple ion bonds of hundreds of ion pairs are required according to our previous results ${ }^{16}$. It is relatively difficult for such long chain segments parallel to each other only by thermal fluctuation. But pre-stretch can substantially increase this process and thus enhance strong bonds formation. While weak bonds are just consisted of several or tens of ion pairs ${ }^{16}$, which is much easier to be formed by thermal fluctuation. Thus, pre-stretch only has a weak effect on weak bonds formation.

Theoretical finite extensibility $\lambda_{\mathrm{m}}$ against $\lambda_{\text {pre }}$ is shown in Figure $7 \mathrm{~d}$. $\lambda_{\mathrm{m}}$ decreases with increasing $\lambda_{\text {pre }}$, in agreement with experimental results of fracture strain $\varepsilon_{\mathrm{b}}$ in Figure 3d. The decrease in $\lambda_{\mathrm{m}}$ should be ascribed to the increase in strong bonds density by 
imposing the pre-stretch. The strong bonds act as quasi-permanent crosslinking, so increasing strong bonds density by pre-stretch leads to a decrease in finite extensibility. The simulated Deborah numbers $D_{\mathrm{e}}$ at different pre-stretch ratio are within the range of the subunit, in agreement with our reported work ${ }^{17}$. For clarity, the data are not presented here.

The pre-stretch method used in PA hydrogels in this study shares analogy to the mechanical stimuli approach used in metalworking ${ }^{28,29}$ or semicrystalline polymer processing ${ }^{30,31}$, which is actually stress or strain induced molecular/atom orientation and thus influences the structure of materials. In metalworking, metal stock is passed through one or several pairs of rollers to decrease the thickness and increase the strength. In semicrystalline polymer processing, shear or extensional flow is used to stretch polymer chains, which leads to the orientated crystals and enhanced performance. Similar to semicrystalline polymers, the physical hydrogel was demonstrated to has facile reprocessability recently ${ }^{32,33}$. That is to say physical hydrogels are promising to be processed with industrial method such as injection modeling or with microfabrication method such as 3D/4D printing, where the stretch of polymer chains is inevitably involved. Therefore, this work provides fundament for future processing of PA or other physical hydrogels.

\section{Conclusion}

In summary, we demonstrated that pre-stretch is an effective method to accelerate the ion complexation dynamics and enhance the final performance of PA hydrogels. The enhancement in performance is attributed to the pre-stretch induced strong bonds formation. Since the origin of structure induced by pre-stretch is ascribed to the entropy decrease and chain alignment increase, this method should not be specific for PA systems but should be 
generic to other physical hydrogels. Therefore, this work will open a new avenue to tailor performance of physical hydrogels as structural biomaterials or industrial materials used in aqueous environment.

\section{Acknowledgements}

This research was partially supported by Grant-in-Aid for Scientific Research (S) (No. 124225006) and for Postdoctoral Fellowships for Foreign Researchers (No. 15F15348) from the Japan Society for the Promotion of Science (JSPS). This research was also partially funded by ImPACT Program of Council for Science, Technology and Innovation (Cabinet Office, Government of Japan).

\section{Supporting Information}

The Supporting Information is available free of charge on the

Figure S1 shows the schematic of experimental procedure.

Figure S2 shows the pre-stretch ratio dependence of water content of PA hydrogels at free state.

Figure S3 shows the wide-angle X-ray scattering (WAXS) measurement of PA hydrogels at free state.

Figure S4 shows the change in orientation of PA hydrogels at free state from WAXS measurement. 
Figure S5 shows the evolution of sample dimension with time in salt solution of PA hydrogels at free state.

\section{References}

1 K. Yasuda, N. Kitamura, J. P. Gong, K. Arakaki, H. J. Kwon, S. Onodera, Y. M. Chen, T. Kurokawa, F. Kanaya, Y. Ohmiya and Y. Osada, Macromol. Biosci., 2009, 9, 307-316.

2 A. S. Hoffman, Adv. Drug Deliv. Rev., 2012, 64, 18-23.

3 D. Seliktar, Science, 2012, 336, 1124-8.

4 R. Jin and P. J. Dijkstra, in Biomedical Applications of Hydrogels Handbook, 2010, vol. c, pp. 203-225.

5 M. Hamidi, A. Azadi and P. Rafiei, Adv. Drug Deliv. Rev., 2008, 60, 1638-1649.

6 T. Baumberger, C. Caroli and D. Martina, Nat. Mater., 2006, 5, 552-5.

7 Y. Tanaka, K. Fukao and Y. Miyamoto, Eur. Phys. J. E. Soft Matter, 2000, 401, $395-401$.

8 J. P. Gong, Science (80-. )., 2014, 344, 161-162.

9 J. P. Gong, Y. Katsuyama, T. Kurokawa and Y. Osada, Adv. Mater., 2003, 15, $1155-$ 1158.

10 M. A. Haque, T. Kurokawa and J. P. Gong, Polymer (Guildf)., 2012, 53, 1805-1822.

11 J. P. Gong, Soft Matter, 2010, 6, 2583.

12 Q. Chen, L. Zhu, H. Chen, H. Yan, L. Huang, J. Yang and J. Zheng, Adv. Funct. Mater., 2015, 25, 1598-1607. 
13 H. J. Zhang, T. L. Sun, A. K. Zhang, Y. Ikura, T. Nakajima, T. Nonoyama, T. Kurokawa, O. Ito, H. Ishitobi and J. P. Gong, Adv Mater, 2016.

14 F. Luo, T. L. Sun, T. Nakajima, T. Kurokawa, Y. Zhao, K. Sato, A. B. Ihsan, X. Li, H. Guo and J. P. Gong, Adv Mater, 2015, 27, 2722-2727.

15 J. Y. Sun, X. Zhao, W. R. Illeperuma, O. Chaudhuri, K. H. Oh, D. J. Mooney, J. J. Vlassak and Z. Suo, Nature, 2012, 489, 133-136.

16 T. L. Sun, T. Kurokawa, S. Kuroda, A. Bin Ihsan, T. Akasaki, K. Sato, T. Nakajima, J. P. Gong, M. A. Haque, T. Nakajima, J. P. Gong and M. A. Haque, Nat. Mater., $2013,12,932-937$.

17 T. L. Sun, F. Luo, T. Kurokawa, S. N. Karobi, T. Nakajima and J. P. Gong, Soft Matter, 2015, 11, 9355-9366.

18 A. E. English, S. Mafé, J. a. Manzanares, X. Yu, A. Y. Grosberg and T. Tanaka, J. Chem. Phys., 1996, 104, 8713.

19 M. Fujine, T. Takigawa and K. Urayama, Macromolecules, 2015, 48, 3622-3628.

20 R. H. Pritchard and E. M. Terentjev, Polym. (United Kingdom), 2013, 54, 6954 6960.

21 N. Bouklas and R. Huang, Soft Matter, 2012, 8, 8194.

22 A. Konda, K. Urayama and T. Takigawa, Macromolecules, 2011, 44, 3000-3006.

23 F. Luo, T. L. Sun, T. Nakajima, D. R. King, T. Kurokawa, Y. Zhao, A. Bin Ihsan, X. Li, H. Guo and J. P. Gong, Macromolecules, 2016, 49, 2750-2760.

24 T. Wang, D. Liu, C. Lian, S. Zheng, X. Liu and Z. Tong, Soft Matter, 2012, 8, 774.

25 D. C. Lin, J. F. Douglas and F. Horkay, Soft Matter, 2010, 6, 3548. 
26 R. E. Webber, C. Creton, H. R. Brown and J. P. Gong, Macromolecules, 2007, 40, 2919-2927.

27 F. Deplace, M. Rabjohns, T. Yamaguchi, A. Foster, C. Carelli, C.-H. Lei, K.

Ouzineb, J. Keddie, P. Lovell and C. Creton, Soft Matter, 2009, 5, 1440-1447.

28 W. J. Arbegast, Hot Deform. Alum. Alloy., 2003, 3, 313.

29 M. H. G. Kuijpers, Beyond Elit. Altern. to Hierarchical Syst. Model. Soc. Form., 2012, 215, 413-421.

30 K. Cui, L. Meng, Y. Ji, J. Li, S. Zhu, X. Li, N. Tian, D. Liu and L. Li, Macromolecules, 2014, 47, 677-686.

31 K. Cui, D. Liu, Y. Ji, N. Huang, Z. Ma, Z. Wang, F. Lv, H. Yang and L. Li, Macromolecules, 2015, 48, 694-699.

32 F. Luo, T. L. Sun, T. Nakajima, T. Kurokawa, A. Bin Ihsan, X. Li, H. Guo and J. P. Gong, ACS Macro Lett., 2015, 4, 961-964.

33 J. Wei, J. Wang, S. Su, S. Wang, J. Qiu, Z. Zhang, G. Christopher, F. Ning and W. Cong, RSC Adv., 2015, 5, 81324-81329. 